\title{
Roles de la comunicación organizacional en la gestión del conocimiento: oportunidades y desafíos a partir de experiencias de grandes empresas antioqueñas
}

\section{Organizational communication roles in knowledge management: based on Antioquia large company's experiences opportunities and challenges}

Doris Elena Muñoz Zapata (Colombia)

Magíster en Comunicación y Educación Universidad Pontificia Bolivariana doris.munoz@upb.edu.co

\section{Resumen}

Se realizó un estudio múltiple de casos, constituidos por veinte empresas grandes del departamento de Antioquia, que fueron analizadas por sectores: servicios, financiero, manufactura, salud, moda y textiles, servicios públicos y construcción. Se analizaron categorías como las características del proceso de gestión de conocimiento, la comunicación interna, la

\author{
Juan Esteban Valencia Rey (Colombia) \\ Magíster en Comunicación Organizacional \\ Universidad Pontificia Bolivariana \\ juanesteban.valencia@upb.edu.co
}

\begin{abstract}
A multiple case study was carried out, comprising twenty Antioquia department large companies, which were analyzed by sectors: services, financial, manufacturing, health, fashion and textiles, public services and construction. Categories such as knowledge management process characteristics, internal communication,
\end{abstract}

Recibido:

Evaluado:

Aceptado:
28 de septiembre de 2016

5 de mayo de 2017

11 de agosto de 2017

\section{PARA CITAR ESTE ARTÍCULO/TO CITE THIS ARTICLE}

Muñoz Zapata, D. E. \& Valencia Rey, J. E. (2017).

Roles de la comunicación organizacional en la gestión del conocimiento: oportunidades y desafíos a partir de experiencias de grandes empresas antioqueñas, Poliantea 13(24), p. 125-140. 
apropiación de los medios de comunicación y los roles que asignan las organizaciones a la comunicación organizacional en su gestión del conocimiento con públicos internos. Predominó en la valoración de las empresas, un rol centrado en la difusión de información, más que una visión estratégica de la comunicación, principalmente en las organizaciones que llevan menos tiempo gestionando conocimiento. También se describen aspectos que dificultan la adecuada gestión del conocimiento, como es la cultura organizacional, las competencias del recurso humano y el desconocimiento frente al tema. Se concluye que predomina una limitada visión frente a las posibilidades de aporte de la comunicación organizacional y la necesidad de articularla estratégicamente en cada una de las etapas de la gestión del conocimiento en las organizaciones.

Palabras Clave: comunicación organizacional, comunicación estratégica, gestión del conocimiento, TIC. media appropriation and organizational communication assigned roles in internal knowledge management were analyzed. In company's valuation, a focused-on dissemination of information role predominated, rather than a communication strategic vision, mainly in organizations that have been less time managing knowledge. It also describes aspects that hinder a proper knowledge management, such as organizational culture, human resources competencies, and ignorance in the matter. It is concluded that a limited vision predominates over contribution possibilities of organizational communication and the need to strategically articulate it in every stage of organizations' knowledge management.

Keywords: organizational communication, strategic communication, knowledge management, $\mathrm{TIC}$. 


\section{Introducción}

La gestión del conocimiento constituye un soporte indispensable para la consolidación de este como un valioso activo intangible, que aporte al crecimiento y sostenibilidad de las organizaciones, en la medida en que permite generar nuevo conocimiento, para el desarrollo de la empresa y la resolución de problemas del entorno (Martínez, 2006). En el caso de las empresas colombianas que libran la batalla por la competencia en mercados globalizados, la adecuada gestión de sus conocimientos, se constituye en una oportunidad para generar valor e innovaciones que fortalezcan sus capacidades de interacción y permanencia en los mercados globales.

Los procesos que conlleva en sí la gestión del conocimiento están fuertemente atravesados por la comunicación, desde sus perspectivas de interacción, relacionamiento, negociación e incluso difusión de informaciones acerca de los conocimientos generados y los procesos que se llevan a cabo en la gestión de este intangible.
Desde un nivel más básico la comunicación organizacional a partir de técnicas y actividades aporta a las necesidades de información de la empresa, difundiendo lo que es y hace la organización (Fernández, 2005), lo cual en términos de gestión de conocimiento se constituye en un componente importante para el flujo de la información que se genera en los procesos de gestión del conocimiento. Sin embargo, los desafíos internos que involucra dicha gestión, requieren una comprensión más estratégica de la comunicación, tal como lo plantea Van Riel (2012), al afirmar que "se espera que los profesionales de la comunicación aumenten el conocimiento y el reconocimiento de la organización $y$, como consecuencia lógica aumenten y mantengan el apoyo a la organización" (Van Riel, 2012). Dicho apoyo, es necesario para obtener una óptima gestión del conocimiento, en la que se requiere que las personas se involucren en profundidad, para tomar conciencia de los conocimientos adquiridos, exteriorizarlos, socializarlos e incluso generar nuevos saberes, a partir de la interacción de los diversos conocimientos compartidos. 
En ese contexto, surgió el proyecto: "Roles de la Comunicación organizacional en la gestión del conocimiento en empresas Antioqueñas" realizado por el grupo de investigación Gestión de la Comunicación -Gescom- de la Facultad de Comunicación Social de la Universidad Pontificia Bolivariana, que buscó aproximarse a los roles asignados a la comunicación organizacional, en empresas de la región, para explorar potencialidades de la comunicación y los comunicadores frente a la gestión del conocimiento, las cuales pueden constituirse en insumo para el fortalecimiento de capacidades y competencias en la formación de los profesionales de la comunicación en programas de pregrado $y$ posgrado.

El estudio se ocupó de las empresas grandes del departamento de Antioquia, en consideración de que representan el $80 \%$ de las exportaciones realizadas por dicha región (ANIF, 2015) y aportaron en 2014 el 19\% del ingreso del país, pese a que tan solo constituyen $0.73 \%$ del total de empresas de esta región.

\section{Marco teórico}

La gestión del conocimiento es una disciplina que parte de la administración, orientada a mejorar el proceso que sufre el conocimiento en una organización (Firestone \& McElroy, 2003). No obstante, el conocimiento sólo es gestionable en la medida en que se gestione su proceso de creación e intercambio, pero no el conocimiento en sí mismo (Arboníes, 2006). Por ello, la gestión del conocimiento está orientada a la "creación y mantenimiento de un sis- tema orgánico, total y unificado, produciendo, manteniendo, mejorando, adquiriendo y transmitiendo el soporte del conocimiento de la empresa" (Firestone \& McElroy, 2003, pág. 71). Por ello, la relación y su diferencia entre información y conocimiento potencialmente redirecciona la gestión del conocimiento según la importancia atribuida. Award y Ghaziri (2004) comprende la información como una forma de moldear los datos para llegar a una elucidación contextual sobre el evento o serie de eventos en una realidad. PérezMontoro (2008) facilita la definición al afirmar que la información es datos que se han estructurado a partir de una clave de sistematización para ser comprendidos por personas que conozcan esa clave. Pero esto no significa que la información sea relevante o tenga sentido tanto para quien la tenga como para quien la puede recibir. El sujeto cognoscente aparece como relevante, ya que es él quien valora la información. Llega a ser conocimiento cuando esa información se suma a otra información que el sujeto cognoscente posee, dotando de sentido esos flujos de información que le permiten comprenderse y comprender el mundo que le rodea. Esto concuerda con los planteamientos de Nonaka y Takeuchi (1999) que el conocimiento está ligado a la acción y experiencia humana, en un contexto social, en el que sólo puede ser creado por los individuos. Justamente la necesidad de escenarios sociales en donde se desarrolle el conocimiento, abre la pregunta por el papel de la comunicación en la gestión del conocimiento. Nonaka y Takeuchi (1999) mencionan que el conocimiento requiere de un alto grado relacional para la construcción de sig- 
nificado en contextos determinados a partir de la información.

Existen diferentes modelos de gestión del conocimiento, de los cuales se revisaron cinco (Kaplan \& Norton, 1992; Demarest, 1997; Nonaka I., 1994; McAdam \& McCreedy, 1999; McAdam, 2000; CEN Work Agreement, 2004), buscando comprender tanto los elementos tenidos en cuenta como la relación que hay con la comunicación y su gestión.

El modelo presentado por Norton y Kaplan (1992) como el Balance Scorecard fue creado inicialmente como un sistema de medición que unificara la información precisa de la organización para la toma de decisiones. Pero además evidenciaba la coherencia y consistencia de la medición frente la estrategia organizacional. Esto permitió que los autores ensancharan su utilidad de tal manera que se pudiera evaluar la coherencia de los medios elegidos con los fines de la organización, las fortalezas de los equipos y procesos por su conocimiento, así como el alcance de la implementación de los objetivos organizacionales en los niveles estratégicos, tácticos y operativos. Este modelo realiza su medición desde cuatro ámbitos: la financiera, los clientes, los procesos internos, y el aprendizaje y el crecimiento. Cada ámbito debe fundarse en la visión y la estrategia organizacional propuesta e influir en los demás ámbitos (Kaplan \& Norton, 1996). Este modelo es uno de gestión del conocimiento porque pretende alinear la organización de acuerdo tanto a la estrategia organizacional elegida como al conocimiento que la organización posee mediante cu- atro procesos: clarificar la visión, comunicar y conectar, planear el negocio y la realimentación y el aprendizaje. Entendiendo que esos procesos se dan en un ciclo en espiral (Kaplan \& Norton, 1996). El interés por este modelo surge de su orientación al conocimiento en lo estratégico, pero por su paradigma altamente administrativo no puntualiza los puntos críticos del ciclo de vida de la gestión del conocimiento. En cuanto a la comunicación, se evidencia que su papel va más allá de una perspectiva difusionista, ya que se orienta a enlazar y conectar, pero esto no precisado en la práctica.

El modelo de Demarest (1997) se acerca a la gestión del conocimiento desde una mirada organizacional buscando integrar implícitamente una mirada social sin dejar claro su aporte. Este modelo tiene cuatro momentos: la construcción del conocimiento, su materialización, la diseminación del conocimiento y su uso. Lo interesante del modelo es la relación que establece entre ellos: La construcción de conocimiento se materializa, pero esa materialización conlleva a la diseminación del conocimiento a otros para su uso. Pero el uso del conocimiento fortalece la materialización, la diseminación y la construcción. Por ello, son procesos interdependientes. McAdam y McCreedy (1999) modificaron el modelo para evidenciar los dos tipos de paradigma que estaban en el modelo, el administrativo y social, a través de los beneficios del negocio y la emancipación de los empleados como resultado del uso del conocimiento. A pesar que se relaciona la gestión del conocimiento con la innovación en el que la primera actúa como catalizadora de la segunda (McAdam, 2000), carece 
de aplicabilidad para los contextos organizacionales altamente pragmáticos de las organizaciones.

El Comité Europeo para la Estandarización (CEN, en sus siglas en francés) realizó un marco de actuación para la gestión del conocimiento en el que se buscaba comprender integralmente el fenómeno. Se divide en tres niveles: El primer nivel se refiere al enfoque del negocio donde se encuentra los procesos de valor del negocio como productos, servicios, clientes y tecnologías, así como las redes del negocio en el que se encuentran. El segundo nivel se refiere a las actividades esenciales para la gestión del conocimiento que se refiere al ciclo de vida de la gestión del conocimiento (identificar, crear, almacenar, compartir y usar) que debe insertarse en la vida cotidiana de la organización. El último nivel se refiere a los habilitadores de la gestión del conocimiento que están divididos en las capacidades de conocimiento personal que describe a aquellas que las personas dentro de la organización poseen o deben desarrollar y las capacidades de conocimiento organizacional, aquellas que se piden en los procesos organizacionales para el funcionamiento adecuado de la organización.

Este modelo cumple lo que promete, ya que tiene en cuenta muchísimos factores organizacionales y los relaciona, buscando la unificación. Sin embargo, su perspectiva altamente administrativa opaca la mirada social que existe en el modelo.

El modelo de Nonaka y Takeuchi (1999) es uno de los primeros modelos que surgieron y que ha influenciado ampliamente otros modelos por su perspectiva única. No obstante, ha sido poco comprendido su aporte desde la perspectiva relacional. Parte de esta incomprensión reside en la reducción del modelo a la generación de conocimiento, sin descubrir en él cómo es posible que lleve el ciclo de vida completo del conocimiento.

El modelo está basado en la propuesta de los tipos de conocimiento tácito y explícito de Polanyi (1966). Está cimentado sobre la base conceptual que los individuos, en un contexto social, sólo los únicos creadores del conocimiento, por lo que la organización por sí misma no puede generarlo. Por ello, el modelo propone cuatro momentos de encuentro de los individuos en el que se convierte el conocimiento tácito a explícito o viceversa, a partir de grupos intraorganizacionales que poco a poco van expandiendo ese conocimiento en el relacionamiento con otros grupos en la compañía a través de esos mismos momentos en forma de espiral. Cada escenario relaciona a los individuos con su contexto social de manera única que impulsa la creación del conocimiento y su utilización. El escenario de socialización busca generar una interacción social en el que se comparta los códigos culturales de la organización como los de los individuos a partir de las relaciones sociales, de sujeto a sujeto, de conocimiento tácito a tácito. La exteriorización o externalización es un escenario de diálogo y reflexión grupal en el que el conocimiento tácito pasa al explícito ante la codificación del conocimiento individual y 
subjetivo mediante el lenguaje que lo hace comprensible y explicable de forma empírica. El escenario de la combinación busca profundizar sistemáticamente en lo generado en el escenario anterior mediante la sistematización de los diferentes conocimientos explícitos buscando la armonía entre ellos (coherencia y cohesión). Es la integración del conocimiento de manera formal en el que hay educación, formación y capacitación del grupo mismo que armoniza el conocimiento. Y el último escenario llamado interiorización que busca llevar a la práctica ese conocimiento formalizado y armonizado a través del quehacer. Es el aprendizaje personal que se tiene al asumir una nueva perspectiva de algo en el que cada individuo vuelve experiencia ese conocimiento, armonizándolo con otras experiencias personales que tiene. Estos escenarios permiten una espiral del conocimiento que pasa de individuos a grupos y de grupos a organizaciones a través de la interacción social.

La comprensión del conocimiento que este modelo tiene como sentido dado por los sujetos cognoscentes, en contextos sociales y nacido de relaciones evidencian el vínculo entre el conocimiento y el tejido social como potenciador del conocimiento. Con ello, no reduce la gestión del conocimiento simplemente a procesos administrativos, sino que toma en cuenta las dinámicas humanas y sociales para que el conocimiento surja de ellas (Nonaka, 1994; Nonaka \& Takeuchi, 1999; Nonaka, Toyama, \& Konno, 2000; Nonaka, Toyama, \& Byosière, 2001).
En todos los modelos, de manera implícita, está presente la comunicación. Sin embargo, la aproximación a ella varía de modelo a modelo. En el modelo de Demarest (1997) con las modificaciones de McAdam \& McCreedy (1999) la comunicación se entiende desde una mirada difusionista, es decir, transmitir la información de tal manera que llegue a usarse. En el modelo de CEN (2004), la comunicación está de manera difusionista en el nivel de las actividades esenciales de la gestión del conocimiento en la actividad de compartir el conocimiento, así como en las redes de los grupos de interés en el nivel del enfoque del negocio, en el que las relaciones públicas son una estrategia clave de manejo comunicativo. En el Balance ScoreCard (Norton \& Kaplan, 1992), la comunicación tiene una perspectiva difusionista $y$, aunque intentan incluir una perspectiva relacional, en la práctica no lo logran. El modelo de Nonaka \& Takeuchi (1999) asume la comunicación implícitamente desde un enfoque relacional y estratégico, ya que en últimas orienta las relaciones hacia la innovación (Nonaka \& Takeuchi, 1999; Esterhuizen, Schutte, \& du Toit, 2012) en pro de la sostenibilidad de la organización.

La comunicación como fenómeno en las organizaciones siempre está presente en las personas, los procesos, las estructuras y la cultura de la compañía. No obstante, este fenómeno ayudará o inhibirá procesos dependiendo de la orientación que se le de. Por ello, la comunicación será estratégica cuando oriente los vínculos con los públicos con los que se relaciona la empresa para generar relaciones duraderas que se conviertan 
en ventajas competitivas (Tironi \& Cavallo, 2006) buscando el logro de los objetivos de la organización (Pizzolante, 2009; Preciado \& Guzman, 2011). Esto quiere decir que la comunicación no sólo se encarga de la adecuada transmisión de información (difusión), sino de una forma de gestión en el que las estrategias den cohesión y coherencia a la gestión organizacional para el cumplimiento de las metas de cada una de las partes para el funcionamiento organizado y consistente de la organización (Pizzolante, 2009).

\section{Metodología}

Se realizó un estudio exploratorio de casos múltiples con el propósito de describir los roles de la comunicación organizacional en los procesos de gestión del conocimiento en grandes empresas del departamento Antioquia.

Las veinte empresas participantes se agruparon por sectores de acuerdo a la actividad principal a la que se dedican, obteniendo en total 7 casos: sector servicios (compuesto por 5 empresas), sector financiero ( 3 empresas), manufactura (3 empresas), servicios públicos (3 empresas), sector servicios de salud (3 organizaciones), construcción (1 empresa) y sector moda-textil (3 empresas).

El estudio respondió a las categorías de análisis: Gestión del conocimiento, comunicación organizacional y gestión del conocimiento, uso y apropiación de medios de comunicación interna.

\section{Criterios de inclusión de la muestra}

Se seleccionaron empresas que, de acuerdo a la Ley 905 de 2004 de la República de Colombia, están clasificadas como grandes empresas, además de tener en ejecución el programa o proceso de gestión del conocimiento, con una antigüedad mínima de un año, que permitiera revisar los roles de la comunicación organizacional y sus equipos de comunicación frente a dichos procesos.

\section{Instrumentos}

Considerando que en el método de estudio de caso los datos pueden obtenerse a partir de diversas fuentes que involucren lo cualitativo y lo cuantitativo (Martínez, 2006), se utilizaron dos instrumentos: una encuesta y una entrevista semiestructurada. La encuesta contó con 11 preguntas, concentradas en las dimensiones de análisis. La entrevista se realizó con 10 preguntas semiestructuradas que fueron respondidas por 11 organizaciones participantes.

Se realizaron 3 aplicaciones piloto para validar la comprensión de las preguntas y los conceptos indagados.

\section{Resultados}

Se realizó la investigación en 7 sectores diferentes: servicios, servicios públicos, financiero, manufacturero, construcción, servicios de salud y modatextil. Cuando se les consultó a las empresas sobre el tiempo de existencia en el mercado, se encontró que tienen un rango que va desde los 19 a los 100 años. 
En las diferentes empresas que constituyen los casos, se indagó por el modelo de gestión de conocimiento utilizado, encontrando que en todas han creado su propio modelo, adaptado a características de la empresa e insertado en el modelo de gestión de la empresa.

Al consultar sobre la importancia que la empresa le otorga a la gestión del conocimiento, el sector manufacturero la considera poco importante, mientras que el sector de servicios públicos no sólo la considera muy importante, sino que además es el sector que más más tiempo lleva en la implementación del proyecto de gestión del conocimiento con una solidez como sector. El sector financiero, construcción y el de servicios también la consideran muy importante. El sector de servicios de salud y modatextil la consideran importante.

En la indagación por la utilización de estrategias de comunicación en el proyecto de gestión del conocimiento, se respondió que sí en todos los sectores. Pero al preguntarles por las áreas o departamentos de comunicaciones, se encontró que no siempre se cuenta con un departamento o área de comunicaciones en las organizaciones; aunque en la mayoría de los sectores existe. Y si existe, no siempre apoya los procesos del proyecto. El caso más evidente lo tiene el sector manufacturero en el que no hay áreas de comunicación y que si lo hay en una empresa, no apoya el proceso de gestión del conocimiento.

En cuanto a la indagación por el rol que juega el área de comunicaciones en las empresas con respecto al proyecto institucional de gestión del conocimiento, la mayoría de las empresas respondió al rol de difundir información del equipo de gestión del conocimiento por medios internos. No obstante, el sector modatextil, de servicios públicos y financiero agregaron que además de ello, el área de comunicaciones planifica pensando en la gestión del conocimiento. En los sectores de construcción y de servicios de salud, además de difundir y apoyar la gestión del conocimiento, el área de comunicaciones participa en la planificación del proyecto de gestión del conocimiento junto con el equipo encargado del tema. Esto es consistente con lo obtenido por las entrevistas.

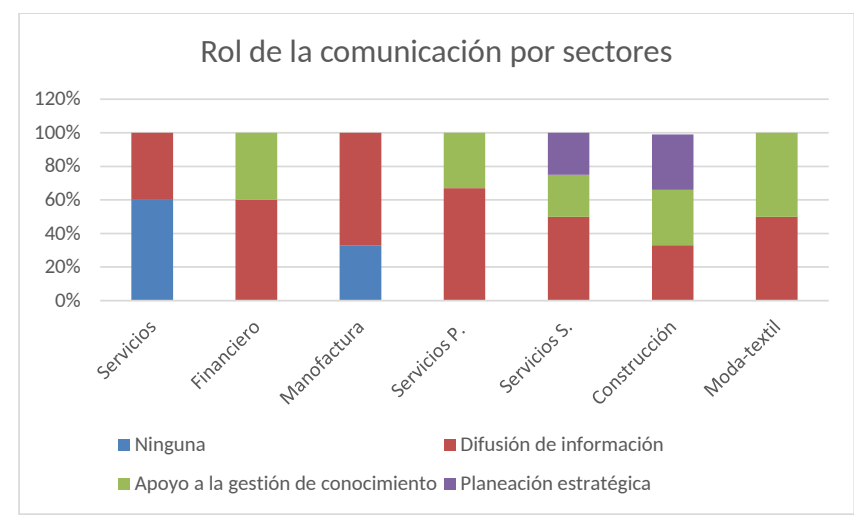


Con respecto a la consulta sobre las formas de gestionar el conocimiento, las empresas contestaron que tanto las reuniones para la documentación de mejoras, nuevas ideas y nuevo conocimiento son las que más utilizan en los diferentes sectores, seguidas de la documentación del conocimiento en los puestos de trabajo. Las plataformas tecnológicas también son una opción de gestión del conocimiento utilizadas por la organización, así como estímulos para los empleados que lo gestionen. El sector de servicios de salud es el que combina la mayoría de las formas de gestión del conocimiento antes mencionadas, seguido por el sector financiero, de servicios públicos y manufacturero.

Al indagar por dificultades en la transferencia de conocimiento, se reportó que en la mayoría de los sectores las hay, a excepción del sector construcción. Las empresas identifican como las fuentes de esas dificultades en la mayoría de los sectores al desconocimiento del tema, la plataforma tecnológica y la cultura organizacional. El sector moda-textil argumenta que son la plataforma tecnológica y la cultura organizacional, mientras que el sector de servicios públicos respondió que además de esas dos opciones, las competencias del recurso humano de la organización y bajo compromiso de los directivos con el tema. Cuando se les pidió argumentación al respecto, se centraron en dos temas: plataformas tecnológicas y personal para el proyecto de gestión del conocimiento. Con respecto a las plataformas tecnológicas, se refieren tanto al uso y apropiación que le dan los empleados como a la adecuación de las plataformas para la gestión del conocimiento. Con respecto al personal, se refieren tanto a la capacitación del mismo como a mayor personal para el equipo.

En cuanto a los medios de comunicación usados para la difusión y la transferencia del conocimiento son distintos dependiendo de los públicos a los que se dirigen, según las empresas. Al relatar el uso y la apropiación que los colaboradores tienen de ellos, las empresas evidencian el uso por política institucional o por proceso, pero no la apropiación del público como tal de los medios.

En todos los sectores están presentes las tecnologías de la información y la comunicación para la gestión del conocimiento. La mayoría han diseñado sus propias plataformas tecnológicas según sus necesidades y unas pocas han aprovechado aquellas TIC que existen en la internet.

\section{Discusión}

En este estudio de caso múltiple se puede observar un contraste entre los modelos de gestión de conocimiento, propuestos por académicos, en los que no sólo 
se tienen en cuenta los elementos necesarios para una adecuada gestión, sino la relación que deben tener estos elementos. Las empresas, desde de su propio contexto, con sus propias convicciones, asumen la gestión del conocimiento y definen qué modelo de gestión les conviene para su realidad particular. Es relevante que en los modelos teóricos se hace referencia a la comunicación relacionada con el paso transferencia en el ciclo de vida de la gestión de conocimiento e implícitamente en otros ángulos de la comunicación como lo relacional y lo estratégico. Los modelos que utilizan las organizaciones para gestionar el conocimiento, se estructuran en conjunto con sus modelos de gestión. Sin embargo, todos, sin excepción, vinculan a la comunicación como un elemento importante para la transferencia del conocimiento.

Sin embargo, el rol que, en la práctica, se le asigna a la gestión de la comunicación parte desde una perspectiva difusionista, es decir, la gestión está centrada en la diseminación de información al proceso de gestión del conocimiento, revisar flujos de información y generación de contenidos para el proceso. Esta focalización reduce la capacidad de esta área de aportar desde lo social para que los colaboradores se sintonicen con el proyecto de gestión del conocimiento al descubrir no sólo su utilidad, sino su aporte a sus propios puestos de trabajo. Como lo afirma García-Morales, Matías-Reche y Verdu-Jover (2011), "la comunicación abierta y flexible es necesaria para el buen desarrollo del aprendizaje organizacional, mientras que la comunicación no participativa y rígida reprime la capacidad para el aprendizaje organizacional" (García-Morales, Matías-Reche,Verdu-Jover, 2011, p. 155). Esto quiere decir que la comunicación no puede limitarse a la mera difusión de información y, con ello, a una comunicación unidireccional, sino que debe aportar desde el contexto a la construcción de tejido social y relacional para que el conocimiento llegue a producirse y a transferirse según las necesidades. Ello implica el clima y la cultura organizacional. Como lo comentó una de las empresas entrevistadas:

\begin{abstract}
"En el mundo organizacional no es sólo emitir, debe haber retroalimentación, esa es la realidad; las comunicaciones tienen que ser muy bien estructuradas y orientadas a resultados, se necesita no solo una persona que comunique sino que entienda el impacto de sus comunicaciones en los resultados del negocio, es una visión más cosmológica de la organización".
\end{abstract}

Ello implica una gestión de la comunicación que se oriente al aporte a corto, mediano y largo plazo a la estrategia organizacional, apalancando procesos que 
estén orientados a esa estrategia como lo es el conocimiento (base para el desarrollo del negocio).

Además, es interesante que no parece haber una relación directa entre aquellas empresas que han comprendido que la gestión de la comunicación les aporta estratégicamente y el tiempo de constitución del proyecto de gestión del conocimiento, aun cuando parece que aquellas que la ven desde la difusión llevan poco tiempo con el proyecto de gestión.

Sobresale que la gestión del conocimiento es valorada como muy importante por el sector financiero, de construcción, de servicios y de servicios públicos, en el que este último, hay una solidez como sector en la implementación del proyecto. Se destaca que siendo el sector manufacturero que valora poco la gestión del conocimiento, y no tiene área de comunicaciones, los responsables de los procesos señalan como las mayores dificultades la falta de competencias en el recurso humano y la cultura organizacional.

En cuanto a los usos de medios y nuevas tecnologías, se destaca que la creatividad para generar herramientas para la gestión del conocimiento, pero aun así no se evidencia apropiación de esas herramientas por parte del personal. Por ejemplo, una de las empresas mencionó que la intranet era un medio para la gestión del conocimiento, pero sólo podía decir que quienes la usaban, lo hacían muy bien, argumentando que había empleados reacios al medio. Otra empresa sostuvo que la plataforma para la gestión del conocimiento no era el problema, sino que la cultura no termina a adherirse a ese proyecto. Otra comentó que debieron realizar una re-inducción a los empleados para hablarle de la gestión del conocimiento con el fin de impulsar el proceso, ya que como carecían de una plataforma particular para ello, los empleados debían hacerlo a través de las TIC. Esto es consistente con esta afirmación de Martínez Crespo \& Giraldo Marín (2012): "es importante comprender...que en los procesos de Gestión del Conocimiento, la tecnología soporta el 10\% de todo el proceso, mientras que el $70 \%$ está soportado en las personas y un $20 \%$ en los procesos" (Martínez Crespo \& Giraldo Marín, 2012, pág. 170).

Esto va en consonancia con las dificultades que se encuentran en la transferencia de conocimiento en los casos estudiados. Estos pueden ser agrupados en la categoría de paradigmas organizacionales en las personas que las conforman: ya que la falta de competencias en el recurso humano, el bajo interés de los directivos y la cultura organizacional, evidenciadas como fuentes por las empresas, sugieren que la gestión de la comunicación debe 
ser orientada a la preparación del contexto organizacional como base para un buen proyecto de gestión del conocimiento: "la planificación estratégica de la tecnología y la gestión de la innovación debe incorporar una estrategia de comunicación interna efectiva que forme un centro participativo en cualquier plan para llevar a cabo innovación organizacional (Hargie y Tourish, 1996)" (García-Morales, Matías-Reche, Verdu-Jover, 2011, p. 156). Esta estrategia de comunicación interna debe ir alineada con toda la organización para que el proceso de gestión del conocimiento comience sólidamente a través de una base cultural que permita fluir el conocimiento dentro de la estructura organizacional. Para ello se requiere identificar y reorientar aquellas barreras que inhiben o anulan el flujo de comunicación en las organizaciones, de tal manera que el conocimiento fluya hacia dónde es necesitado: "En cualquier sistema de comunicación humano, varias fuerzas se desarrollan e inhiben la transferencia de significado eficaz. El proceso de utilización de conocimiento no es ninguna excepción. Muchas de las barreras del flujo de conocimiento son las mismas como aquellos encontrados en el modelo de comunicación simple interpersonal". (Duncan, 1973, pág. 5).

Esto implica comprender la innovación como el horizonte de la gestión del conocimiento, desde la dinámica de la sociedad (Armando, 2013). Esta dinámica no se refiere sólo a la realidad externa de la empresa, sino a todo el entramado relacional de la organización que es la base para reconocer que "el mejor indicador de la potencialidad innovadora $\mathrm{y} / \mathrm{o}$ de competencia de una empresa es la comunicación, el estado de las relaciones" (Carballo, 2006, p. 20 ).

\section{Conclusiones}

El estudio destaca la presencia y valoración positiva de la comunicación interna de las organizaciones, como un componente que aporta a los procesos de gestión del conocimiento, a través de la utilización de canales de comunicación directa y medios de comunicación soportados en las TIC.

A partir de la revisión bibliográfica y de la valoración de los modelos propios que adaptan las organizaciones para gestionar sus conocimientos, se sugieren roles más estratégicos para la comunicación organizacional que conciban la gestión del conocimiento como un proceso profundo, que requiere convocar, motivar, generar diálogos y encuentros que permitan la apertura de los públicos internos frente a sus saberes propios y los colectivos para una construcción conjunta que conlleve a la generación de nuevos conocimientos e innovaciones. 
Es importante articular las problemáticas que se encuentran las organizaciones en su gestión del conocimiento, como lo son las limitaciones de la cultura organizacional, las limitaciones del recurso humano y el desinterés por parte de los directivos, en la gestión de la comunicación de las empresas que emprenden este tipo de iniciativas.

Por último, se destaca la relevancia de la gestión del conocimiento como temática para el análisis y la discusión en procesos formativos e investigativos de los comunicadores que piensan los contextos organizacionales para sociedades más inteligentes y sostenibles.

\section{Referencias}

Arboníes, Á. L. (2006). Conocimiento para innovar. Madrid: Díaz de Santos.

Armando, M. V. (2013). Innovación y dinámica social. Revista iberoamericana de Argumentación (7), 1-11.

Awad, E. M., \& Ghaziri, H. M. (2004). Knoledge Management. New Jersey: Pearson Education.

Cámara de Comercio de Medellín para Antioquia (s.f.). La gran empresa en Antioquia, importancia y desempeño reciente. [diapositivas de PowerPoint]. Recuperado de Cámara de Comercio de Medellín para Antioquia: www.camaramedellin.com.co/site/Portals/0/Documentos/2016/ Anif/Presentacion\%20ANIF\%20 2015.pdf

Carballo, R. (2006). Innovación y gestión de conocimiento. España: Díaz de Santos.

CEN Work Agreement. (2004). European guide to good practice in Knowledge management - Part 1: knowledge management framework. Brussels: European Commitee for Standardization.

Demarest, M. (1997). Understanding Knowledge Management. Long Range Planning, 30(3), 374-384.

Duncan, W. (1973) International Journal of Business Communication, 11 (1), 3-14.

Dinero, R. (2015). Las Empresas Más Grandes del País por Regiones. [en línea] Revista Dinero. Disponible en: www.dinero.com/edicionimpresa/caratula/articulo/lasempresas-mas-grandes-delpais-regiones-2015/209423 [Revisado 27 Sep. 2016].

Esterhuizen, D., Schutte, C. S., \& du Toit, A. S. (2012). Knowledge creation processes as critical enablers for innovation. Internacional Journal of Information Management(32), 354-364. 
Fernández, C. (2005). La comunicación en las organizaciones. México D.F: Editorial Trillas.

Firestone, J. M., \& McElroy, M. W. (2003). Key Issues in the new knowlegde management. Burtington: KMCI.

García-Morales, V. J., MatíasReche, F., \& Verdu-Jover, A. J. (2011). Influence of Internal Communication on Technological Proactivity, Organizational Learning, and Organizational Innovation in the Pharmaceutical sector. Journal of Communication, 61(1), 150-177.

Kaplan, R. S., \& Norton, D. P. (January-February de 1992). The Balanced Scorecard - Measures that Drive Performance. Harvard Bussiness Review, 7079 .

Kaplan, R. S., \& Norton, D. P. (January-Febraury de 1996). Using the Balanced Scorecard as a Strategic Management System. Harvard Bussiness Review, 7585.

Martínez Crespo, J., \& Giraldo Marín, L. (2012). La organización y su adaptación a las tecnologías de la información y la comunicación en procesos de gestión del conocimiento (Spanish). Semestre Económico, 15(32), 161-184.

Martínez, P. (2006). El método de estudio de caso: estrategia metodológica de la investigación científica. Pensamiento y Gestión, No 20.

McAdam, R. (2000). Knowledge Management as a Catalyst for Innovation within Organizations: A qualitative Study. Knowlegde and Process Management, 7(4), 233-241.

McAdam, R., \& McCreedy, S. (1999). The process of Knowledge Management within organizations: a Critical assesstment of both theory and Practice. Knowledge and Process Management, 6(2), 101-113.

Nonaka, I. (1994). A dynamic theory of Organizational Knowledge Creation. Organization Science, 5(1), 14-37.

Nonaka, I., \& Takeuchi, H. (1999). La organización creadora de conocimiento. México: Oxford University Press.

Nonaka, I., \& Takeuchi, H. (1999). La organización creadora de conocimiento. Oxford: University Press.

Nonaka, i., Toyama, R., \& Byosière, P. (2001). A the- 
ory of organizational knowledge creation: understanding the dynamic process of creating knowledge. En M. Dierkes, A. Berthoin Antal, J. Child, \& I. Nonaka, Handbook of Organizational Learning and Knowledge (págs. 491-517). New York: Oxford University Press.

Nonaka, I., Toyama, R., \& Konno, N. (2000). SECI, $\mathrm{Ba}$ and leadership: a unifed model of dynamic knowledge creation. Long range planning(33), 5-34.

Pérez-Montoro, M. (2008). Gestión del conocimiento en las organizaciones: Fundamentos, metodología y praxis. España: Trea.

Pizzolante, I. (2009). De la Re- sponsabilidad Social Empresarial a la Empresa Socialmente Responsable. Madrid: Ediciones de las Ciencias Sociales.

Polanyi, M. (1966). The tacit dimension. New York: Doubleday \& company, inc.

Preciado, A., \& Guzmán, H. (2011) Usos y prácticas de comunicación estratégica en organizaciones públicas y políticas. Folios, Universidad de Antioquia, 25; 49-73.

Tironi, E., \& Cavallo, A. (2006). Comunicación Estratégica. Santiago de Chile: Aguilar.

Van Riel, C. (2012). Alinear para ganar. Madrid: LID Editorial empresarial. 